\title{
Konversion der US-Stützpunkte auf den Philippinen: Kompensationsstrategien in militärdominierten Regionalökonomien
}

\author{
Volker Zimmer, Hamburg
}

\section{Einleitung}

Die Entspannung des Ost-West-Gegensatzes Anfang der 90er Jahre des 20. Jahrhunderts, die schließlich in der Auflösung des Warschauer Paktes mündete, war der Beginn eines tiefgreifenden Umbruchs in den militärischen Komplexen der industrialisierten Staaten. Eine Vielzahl der von den Großmächten beider Seiten weltweit unterhaltenen Stützpunkte verloren in dieser Zeit an strategischer Bedeutung. Die Standorte außerhalb des eigenen Landes wurden insbesondere aufgrund der hohen Kosten immer häufiger in Frage gestellt. Im Jahr 1992 endete auf den Philippinen die Militärpräsenz der USA.

Die Konzentration der auf den Philippinen stationierten US-Streitkräfte auf wenige, sehr große Stützpunkte hatte eine weitgehende Ausrichtung der regionalen Ökonomien auf den konjunkturunabhängigen Wirtschaftsfaktor «Militär» zur Folge gehabt. Der Abzug der US-Streitkräfte und die Schließung der Stützpunkte ließ große Ausfalleffekte erwarten. Es waren schnelle und wirkungsvolle Maßnahmen auf nationalstaatlicher sowie kommunaler Ebene erforderlich, die auf eine Konversion der militärabhängigen Stationierungsregionen abzielten.

Der Begriff Konversion bezeichnet in diesem Sinnzusammenhang «die gezielte Umstellung von vormals für militärische Zwecke genutzten personellen, materiellen und finanziellen Ressourcen auf zivile Zweckbestimmungen» (MeNKE-GlüCKERT 1991 zit. nach WINKELMANN 1995: 15).

\section{Geschichtlicher Abriss}

Der Sieg der USA im Spanisch-Amerikanischen Krieg 1898 beendete die 330-jährige Herrschaft Spaniens über die Philippinen. Doch wurde den Philippinen die erhoffte Unabhängigkeit verwehrt; die USA erwarben das Land im Vertrag von Paris im Jahr 1898. Dies war der Beginn einer massiven US-Militärpräsenz auf den Philippinen, die nur unterbrochen wurde von der japanischen Besatzung während des Zweiten Weltkriegs. Nach der Befreiung durch die USA erhielten die Philippinen zwar ihre staatliche Unabhängigkeit, doch bestanden weiterhin starke wirtschaftliche und militärische Verflechtungen mit den USA. Die US-Truppenpräsenz an insgesamt 23 Standorten wurde 1947 im Military Bases Agreement (MBA) für weitere 99 Jahre festgeschrieben.

Verschiedene juristische Streitigkeiten, insbesondere in Verbindung mit der steigenden Kriminalität amerikanischer Soldaten sowie der Wunsch der Filipinos nach absoluter nationaler Souveränität führten zu einer ganzen Reihe von Änderungen des MBA in den folgenden Jahrzehnten. Die Zahl der Stützpunkte wurde stark reduziert und 1979 wurde die Laufzeit des MBA schließlich verkürzt: Die Stationierung US-amerikanischer Truppen sollte demnach 1991, sofern kein neues Abkommen ausgehandelt würde, enden. Derartige Verhandlungen begannen 1990. Doch die veränderte weltpolitische Situation ließ es für die USA immer weniger sinnvoll erscheinen, die amerikanischen Stützpunkte zu erhalten (CARPENTER 1991). Als im Juni 1991 darüber hinaus noch der Mt. Pinatubo ausbrach und große Bereiche der unweit gelegenen Stützpunkte Subic Naval Base und Clark Air Base zerstörte, gab das amerikanische Verteidigungsministerium Clark Air Base aufgrund des besonders großen Ausmaßes der Schäden auf. Die Verhandlungen zwischen beiden Staaten beschränkten sich nun ausschließlich auf Subic Naval Base und wurden erfolgreich abgeschlossen. Es wurde eine Verlängerung der Stationierung um weitere zehn Jahre vereinbart. Einzig und allein stand noch die Zustimmung des philippinischen Senates aus. Nach langen Diskussionen versagte der Senat die für die Verlängerung notwendige Zweidrittelmehrheit. Infolgedessen mussten bis zum September 1992 alle USamerikanischen Verbände die Philippinen verlassen. Damit endete die Geschichte einer fast hundertjährigen amerikanischen Militärpräsenz auf den Philippinen und es begann der Prozess der Konversion. Es galt jetzt, für die beiden großen Stützpunkte Subic Naval Base (ungefähr $120 \mathrm{~km}$ nordwestlich Manilas), Clark Air Base (ungefähr $80 \mathrm{~km}$ nördlich der Hauptstadt) sowie für die kleineren in Nord-Luzon gelegenen Liegenschaften Camp John Hay in Baguio City und Wallace Air Station in San Fernando (Poro Point) zivile Nachnutzungen zu finden. Die Bemühungen mussten auf eine schnelle, aber auch dauerhafte Kompensation des Ausfalls des für die regionalen Ökonomien äußerst wichtigen Faktors «Militär» ausgerichtet sein (Abb. 1).

\section{Raumwirksamkeit des Wirtschaftsfaktors «Militär»}

Die Verteidigungsetats der Staaten der Erde sum- 


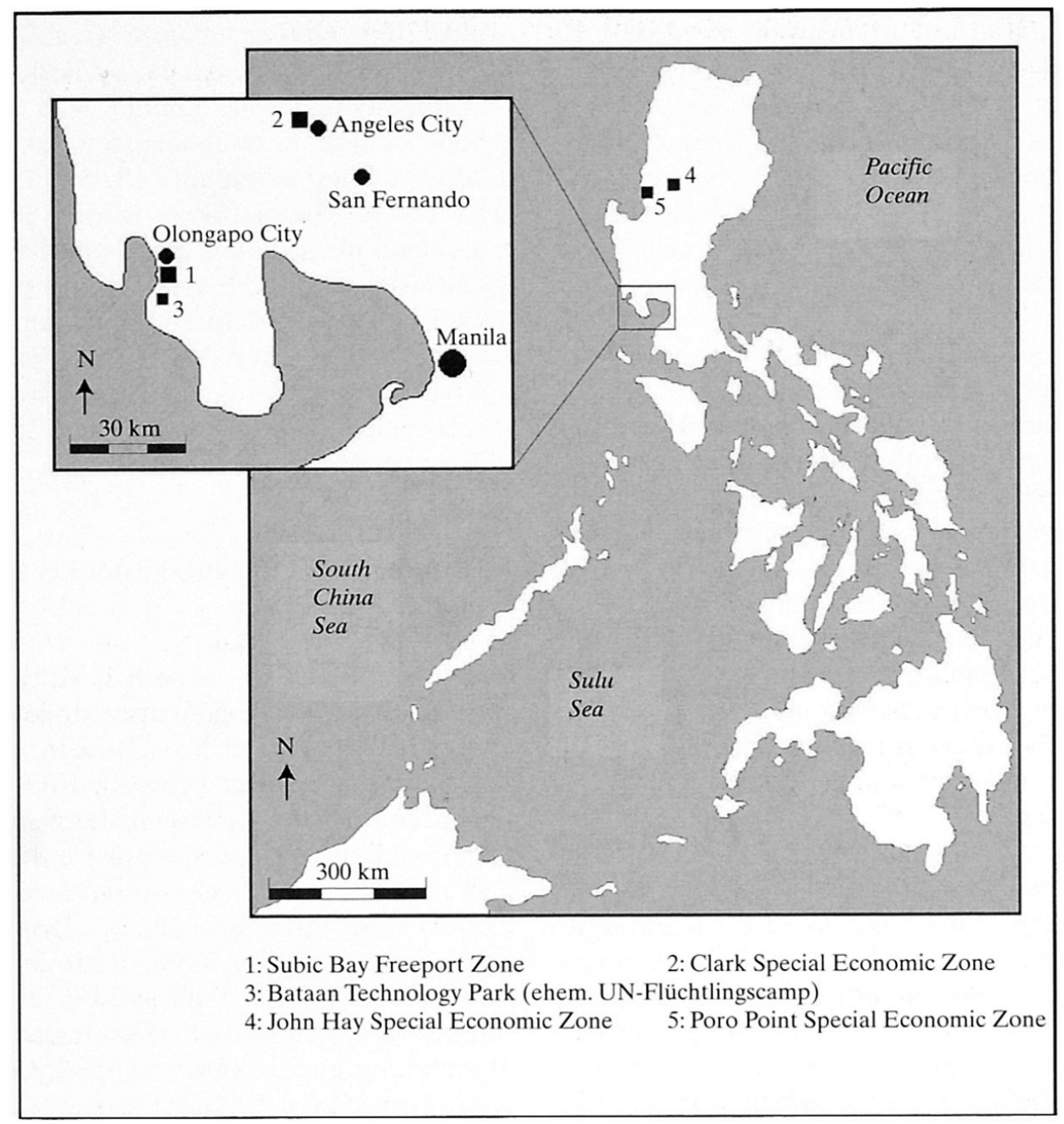

Abb. 1: Ehemalige US-Stützpunkte auf den Philippinen und Konversionsstandorte der Bases Conversion and Development Authority

Former US-military bases in the Philippines and conversion sites of the Bases Conversion and Development Authority

Anciennes bases militaires des Etats-Unis aux Philippines et lieux de conversion de la "Bases Conversion and Development Authority"

Entwurf: V. Zimmer, Kartographie: P. BüchSEL, V. Zımmer; Kartengrundlage: Nelles Maps, Philippines, 1:1,5 Mio.

mierten sich im Jahr 1989 auf fast 1 Billion US\$ weltweit. Am Ende des «Kalten Krieges» wurde mit $85 \%$ (850 Mrd. US\$) ein Großteil hiervon in den Industriestaaten der Nordhalbkugel aufgewandt. Zehn Jahre später ist mit knapp 700 Mrd. US\$ (-30\%) ein massiver Rückgang der weltweiten staatlichen Verteidigungsausgaben zu verzeichnen. Insbesondere in den Industriestaaten wurden die Wehretats deutlich gekürzt und beliefen sich im Jahr 1999 auf nur noch 520 Mrd. US\$ $(-40 \%)$ (Bonn International Center for ConVERSION 2000: 148; basierend auf Preisen und Wechselkursen des Jahres 1993). Im Vergleich zu einem weltweiten Bruttosozialprodukt von rund 30 Billionen US\$ in 1999 wird deutlich, dass auch noch zehn Jahre nach Ende des «Kalten Krieges» knapp 2,5\% der weltweiten Wertschöpfung auf staatliche Militärausgaben zurückzuführen sind.

Die Ausgaben der Verteidigungsetats gliedern sich in eine große Bandbreite von einzelnen Posten auf. Die Betrachtung der räumlichen Wirksamkeit der Militärausgaben zeigt deutlich, wie unterschiedlich die Ausgabeposten in ihrer Bedeutung für die eigentlichen Stationierungsregionen sind (KLÜVER 1987; ROEDER 1989; Biehler, Richter \& STRÄTER 1990):

- Die zentrale Beschaffung von Wehrmaterial, Fahrzeugen und Ausrüstungsgegenständen, die in großer 
Stückzahl standardisiert nachgefragt werden, wird in der Regel ausschließlich auf der nationalen bzw. internationalen Ebene wirksam. Die Zielrichtung der Zahlungsströme konzentriert sich zwar durchaus auf bestimmte, von Wehrtechnik in ihrer Wirtschaftsstruktur geprägte Regionen, doch ist sie für die eigentlichen Stationierungsregionen nicht weiter von Bedeutung.

- Von besonderer Bedeutung für Stationierungsregionen dagegen sind die Personalausgaben für das militärische und zivile Personal. Insbesondere das Zivilpersonal rekrutiert sich in der Regel aus dem regionalen Kontext. Im Fall des militärischen Personals ist dagegen a priori davon auszugehen, dass hier ein Zuzug stattfindet. Soweit es sich um Soldaten mit einer längeren Verpflichtungszeit und mit Familie handelt, ist in der Regel von einer Wohnortwahl in der Nähe des Standortes auszugehen. Die Konsumausgaben werden daher im gleichen Maße wie auch bei den Zivilbeschäftigten vor allem lokal, aber zumindest regional, wirksam.

Nach Ausscheiden aus dem Dienst bleibt ein Teil des militärischen Personals der Stationierungsgemeinde erhalten: Entweder als gut ausgebildete Neuzugänge zum regionalen Arbeitsmarkt oder als Ruheständler mit einem gesicherten Einkommen.

Der Militärstandort sorgt für einen stetigen Bevölkerungszufluss, der nach einiger Zeit zu einem stabilen Fließgleichgewicht auf einem durch die Militärpräsenz erhöhten Niveau der Einwohnerzahl führt.

- Die Nachfrage der Truppe nach Gütern und Dienstleistungen handelsüblicher Art findet in vielen Fällen in einem regionalen Rahmen seine Befriedigung. Daher wird hier auch von der dezentralen Beschaffung gesprochen. Für die Reichweite der Nachfrage ist hier vor allem die Ausstattung der Stationierungsregion mit entsprechenden Angeboten entscheidend.

- Neben den positiven, vor allem ökonomisch begründeten Effekten einer stetigen Militärpräsenz sind allerdings auch negative, besonders im lokalen Kontext spürbare Auswirkungen zu nennen: Die militärische Flächennutzung beeinträchtigt die Entwicklungsmöglichkeiten der Stationierungsgemeinden, da die militärischen Liegenschaften unzugänglich und nicht durchquerbar sind. Städtebauliche Bezüge werden unterbrochen, attraktivere Alternativnutzungen verhindert und für die Entwicklung der Gemeinde notwendige Erweiterungsflächen belegt. Der militärische Übungs- und Ausbildungsbetrieb birgt für die benachbarte Bevölkerung und die Umwelt Risikopotenziale. Es besteht in Stationierungsgemeinden die Gefahr, sich auf den weitgehend konjunkturunabhängigen Faktor «Militär» zu verlassen und damit eine allein auf das Militär ausgerichtete Monostruktur in den Stationierungsgemeinden auszubilden. Im Umfeld von dominanten Garnisonen sind die sozialen und politischen Netzwerke weitgehend durch Mitglieder der Streitkräfte geprägt. Ein hoher Anteil männlicher, junger und unverheirateter Soldaten am Standort fördert zudem Auswüchse des Nachtlebens und insbesondere die Prostitution.

\section{Wirtschaftliche Bedeutung der US-Militärpräsenz auf den Philippinen}

In der im Vorfeld des Abzuges auf den Philippinen geführten Diskussion wurde immer wieder die enorme wirtschaftliche Bedeutung der USMilitärpräsenz betont (Tab. 1). Allein die seit 1979 gezahlten Mieten im Rahmen des Security Assistance Program beliefen sich anfangs auf rund 100 Mio. US\$ pro Jahr und stiegen in der zweiten Hälfte der 1980er Jahre auf mehr als das Doppelte. Die von den USA im Jahre 1988 geleisteten Zahlungen in Höhe von 225 Mio. US\$ stellten ca. 5\% der Einnahmen des philippinischen Staatshaushaltes (eigene Berechnung nach Cranston 1990: GE 39; StatistiSCHES BUNDESAMT 1993: 107).

Die für die Stationierungsregionen aufgrund ihrer räumlich begrenzten Wirksamkeit besonders bedeutenden Personalausgaben summierten sich in der zweiten Hälfte der 1980er Jahre auf ungefähr 280 Mio. US\$ pro Jahr. Auf diesem Höhepunkt der amerikanischen Stationierung befanden sich fast 40.000 Soldaten, Zivilbeschäftigte und Familienangehörige mit US-Pass auf den Philippinen. Viele lebten «offbase» (in Clark: 40\%) und waren daher relativ stark in die lokale Wirtschaft eingebunden (Ausstellung Clark Museum). Darüber hinaus waren die US-Streitkräfte nach dem philippinischen Staat der zweitgrößte Arbeitgeber: Die Zahl der bei der USArmee beschäftigten Filipinos schwankte seit den frühen 70er Jahren zwischen 11.500 und 14.000. Noch 1990 erreichte in Vorbereitung der Intervention «Desert Storm» im Persischen Golf die Zahl der philippinischen Zivilbeschäftigten auf den USStützpunkten mit 14.400 einen letzten Höhepunkt (R. JORSTAD, Statistical Information Analysis Division, Department of Defense, Pers. Mitteilung, März 2001; Department of Defense 1990: 32). Das Niveau der Einkommen, die bei den US-Streitkräften für die einheimischen Arbeitnehmer zu erzielen waren, lag zweibis dreimal höher als die Einkommen des Landes- 


\begin{tabular}{|c|c|c|}
\hline & $\begin{array}{c}\text { Subic } \\
\text { Naval Base }\end{array}$ & $\begin{array}{c}\text { Clark } \\
\text { Air Base } \\
\end{array}$ \\
\hline \multicolumn{3}{|c|}{ 1. Zwischenstaatliche Mietzahlungen (1979 ... 1989) } \\
\hline $\begin{array}{l}\text { Mietzahlungen } \\
\text { für alle Basen }\end{array}$ & \multicolumn{2}{|c|}{$100 \ldots 225$} \\
\hline \multicolumn{3}{|c|}{ 2. Ausgaben im Zusammenhang mit Personal (1987) } \\
\hline $\begin{array}{l}\text { US-Soldaten und US- } \\
\text { Zivilangestellte sowie der } \\
\text { Familienangehörigen }\end{array}$ & 25,1 & 28,4 \\
\hline Soldaten auf Urlaub & 37,2 & 4,0 \\
\hline $\begin{array}{l}\text { Pensioniertes } \\
\text { US-Personal mit Wohnsitz } \\
\text { auf den Philippinen }\end{array}$ & 33,9 & 4,4 \\
\hline $\begin{array}{l}\text { Einrichtungen für das US- } \\
\text { Personal und «off-base»- } \\
\text { Mieten }\end{array}$ & 24,7 & 28,7 \\
\hline $\begin{array}{l}\text { Löhne der philippinischen } \\
\text { Beschäftigten }\end{array}$ & 65,9 & 30,5 \\
\hline \multicolumn{3}{|c|}{ 3. Ausgaben für Güter und Dienstleistungen (1987) } \\
\hline $\begin{array}{l}\text { Verträge mit lokalen } \\
\text { Dienstleistungsunter- } \\
\text { nehmen und der } \\
\text { Bauwirtschaft }\end{array}$ & 53,8 & 27,8 \\
\hline $\begin{array}{l}\text { Material- und } \\
\text { Rohstoffbeschaffung }\end{array}$ & 115,8 & 27,8 \\
\hline \multicolumn{3}{|c|}{ 4. Wohltätige Aussenwirkung der Stützpunkte (1987) } \\
\hline Gemeinnützige Arbeit & 3,3 & 5,7 \\
\hline
\end{tabular}

Tab. 1: Wirtschaftliche Bedeutung der US-Militärpräsenz auf den Philippinen - Ausgaben in Mio. US\$ Economic significance of US military presence in the Philippines - Expenditure in Mio. US\$ Importance économique de la présence militaire américaine aux Philippines - Dépenses en millions de dollars US Quelle: CARPENTER 1991; CRANSTON 1990; Rocher 1990

durchschnitts (eigene Berechnung nach RocHER 1990: 79; Statistisches Bundesamt 1993: 112-115). Eine weitere Personengruppe, die regional, insbesondere im Umfeld von Subic Bay, in Erscheinung tritt, ist die Gruppe der pensionierten US-Militärangehörigen, die hier ihren Alterswohnsitz gefunden haben. Diese Personengruppe ist zwar nicht unmittelbar vom Abzug betroffen, doch ist ein «Nachwachsen» weiterer Pensionärsgenerationen in früherem Umfang auszuschließen.

Die Ausgaben für die dezentrale Beschaffung von Gütern und Dienstleistungen mit einem Volumen von insgesamt 230 Mio. US\$ in 1987 sind nur teilweise der regionalen Wirtschaft zugute gekommen. Der Anteil der Material- und Rohstoffbeschaffung ist tendenziell eher im nationalen und internationalen Rahmen wirksam gewesen, wogegen aber frische Nahrungsmittel und Dienstleistungen eher im lokalen Umfeld nachgefragt worden sind.
Hohe Schätzungen gehen davon aus, dass $80 \%$ aller wirtschaftlichen Aktivitäten in Angeles City direkt oder indirekt auf die benachbarte Clark Air Base zurückzuführen waren. Auf der nationalen Ebene lag die Summe aller Ausgaben des US-Militärs bei durchschnittlich $5,2 \%$ des BSP in den Jahren zwischen 1980 und 1986. Inklusiv aller Sekundäreffekte wird davon ausgegangen, dass die Beschäftigung von rund 68.000 Filipinos direkt oder indirekt durch die USMilitärpräsenz auf den Philippinen induziert wurde (Cranston 1990: GE 34-35). Angeles City nahm aufgrund seiner durch die Militärpräsenz besonderen wirtschaftlichen Dynamik eine Sonderstellung in dem im übrigen extrem unausgewogenen hierarchischen Städtesystem auf der Hauptinsel Luzon ein (BRONGER 1987: 351). Diese Tatsachen machen deutlich, welch hohe Bedeutung das US-Militär für die philippinische Wirtschaft und vor allem für die Ökonomie der Standortregionen hatte. Damit wird aber auch deutlich, welch große Anstrengungen den Philippinen und den Stand- 
ortgemeinden bevorstanden, den Ausfall eines so starken wirtschaftlichen Faktors auszugleichen. Das einzige, was in den Prozess der Konversion eingebracht werden konnte, war eine ursprünglich gut ausgebaute, aber in Teilen nur noch bedingt funktionstüchtige Infrastruktur sowie ein Reservoir gut ausgebildeter, englisch-sprechender einheimischer Arbeitskräfte.

\section{Organisation des Konversionsprozesses}

Die Gestaltung des Konversionsprozesses wurde der durch den Republic Act No. 7227 gegründeten Bases Conversion Development Authority (BCDA) übertragen. Trotz der für philippinische Verhältnisse guten infrastrukturellen Ausstattung der Liegenschaften war absehbar, dass die BCDA immense finanzielle Mittel benötigen würde, um eine auf lange Frist angelegte Entwicklung sicherstellen zu können. Eine Finanzierung dieses Prozesses aus dem laufenden Staatshaushalt war nicht möglich, so dass man sich für einen revolvierenden Fonds entschied, dessen Einnahmen aus dem Verkauf großer Liegenschaften des philippinischen Militärs in Metro Manila generiert werden sollten. Das philippinische Militär unterhielt u.a. mit dem Fort Bonifacio und der Villamor Air Base hier zwei Militärcamps auf besonders wertvollen Liegenschaften in direkter Nachbarschaft zu Makati, dem CBD der Hauptstadtregion, bzw. zum Ninoy Aquino International Airport. Nach Maßgabe des Republic Act sollten 27\% der Verkaufserlöse der BCDA für die Entwicklung der USLiegenschaften zur Verfügung gestellt werden. Größter Nutznießer sollte das philippinische Militär mit einem Anteil von $35,5 \%$ an den Verkaufserlösen sein. Dieses Geld war für die Umsiedlung der militärischen Einrichtungen und die Modernisierung der Ausrüstung vorgesehen.Zur Erleichterung der Investitionstätigkeit wurde den von der Konversion besonders betroffenen Regionen das Privileg einer Freihandelszone eingeräumt. Mit einem weitgehenden Erlass von Steuern, Zöllen auf alle Importe und Exporte, freiem Zugang für ausländische Investitionen, keinen Devisenkontrollen sowie der Vergabe von Dauervisa an Investoren wurden Vergünstigungen gewährt, die denen der 29 Freihandelszonen der Philippine Economic Zone Authority (PEZA) sehr ähnlich sind.

\section{Formen militärischer Flächennutzung und Potenziale der Liegenschaftskonversion}

Militärische Liegenschaften stellen eine Form von Sonderimmobilien dar, deren Nutzung für andere, nicht militärische Zwecke aufgrund der besonderen Beschaffenheit des Geländes und der Gebäude in fast allen Fällen nicht direkt möglich ist.

- Kasernen bestehen in ihrem Kern meist aus einer
Reihe von Unterkunfts-, Stabs- und Wirtschaftsgebäuden, die ergänzt werden durch Fahrzeug-, Lager-, Werkstatthallen sowie teilweise ober- und unterirdische Bunkeranlagen.

- Streitkräfte nutzen für die militärische Lagerhaltung größeren Maßstabs Depots. Auf den meist sehr weitläufigen, naturbelassenen Arealen findet man ober- und unterirdische Bunkeranlagen für die Lagerung von Munition, Treibstoff und Versorgungsgütern.

- Je nach naturräumlicher sowie militärstrategischer Lage behalten sich Streitkräfte eine eigene Verkehrinfrastruktur vor: Häfen, Flugplätze und Pipelines.

- Für den militärischen Übungsbetrieb werden Übungsplätze verschiedener Größenordnung vorgehalten.

Militärischen Liegenschaften weltweit ist gemeinsam, dass sie in aller Regel während der Nutzungszeit unzugänglich sind. Mit dem Abzug der militärischen Einheiten und der Freigabe der Liegenschaften steht den Stationierungsgemeinden eine neue, bisher aus der Ortsplanung ausgeschlossene Fläche frei zur Disposition. Die sich aus dieser Situation ergebenden Potenziale einer Liegenschaftskonversion sind in erster Linie stark abhängig von der wirtschaftlichen Stärke des regionalen Umfeldes. Zumeist erweisen sich anfängliche Vorstellungen einer direkten Anschlussnutzung des militärischen Gebäudebestandes in wirtschaftlicher Form als Illusion. Die Anforderungen an eine dem Standort entsprechende Nutzung ist in den meisten Fällen inkompatibel zu den vorzufindenden ehemaligen militärischen Strukturen. In den Industriestaaten, insbesondere in den wirtschaftlich starken Agglomerationsräumen, werden daher die Flächen vornehmlich von ihrem militärspezifischen Gebäudebestand geräumt und in den Zustand freien Baulands versetzt.

\section{Potenziale und Visionen der Konversion auf den Philippinen}

Die Liegenschaften der US-Streitkräfte auf den Philippinen waren als autarke, umzäunte städtische Siedlungen angelegt. Neben den typisch militärischen Einrichtungen befinden sich hier daneben noch zivile Wohnsiedlungen, Hotels, Versorgungs- und Freizeiteinrichtungen sowie Schulen und Krankenhäuser. Subic Naval Base als ehemaliger Marinestützpunkt mit ausgedehnten Hafenanlagen und Clark Air Base als Luftwaffenstützpunkt mit einem entsprechend den militärischen Anforderungen groß dimensionierten Flugplatz sind in einem besonderen Maße durch ihre militärische Vornutzung geprägt. Insbesondere diese bilden den Kern der Zukunftsvisionen für die Liegenschaftskonversion dieser beiden ehemaligen Großstandorte. Die ehemalige Subic Naval Base ist 
von ihrer Anlage her geeignet, in der Zukunft als Alternative zu dem an seiner Kapazitätsgrenze arbeitenden Hafen Manilas entwickelt zu werden. Die Clark Air Base wird schon heute als Passagier-, aber vor allem als Frachtflughafen genutzt.

\subsection{Mittelfristiges Entwicklungsszenario (10-20 Jahre)}

Die Stärke der beiden großen ehemaligen Stützpunkte wird in einer engen Kooperation gesehen. Der Hafen Subic Bays kann der Clark Special Economic Zone als dringend notwendiger Meereszugang dienen. Doch zur Zeit stellt die schlechte Verkehrsverbindung sowie die bisher unzureichende Ausstattung des Hafens von Subic einen bedeutenden Engpass dar. Die Kapazität ist aufgrund fehlender Containerbrücken stark eingeschränkt und liegt weit hinter der Manilas zurück. Dieser limitierende Faktor soll in den nächsten Jahren durch die Installation neuer Containerumschlagseinrichtungen und den Bau eines tollway zwischen dem Subic Bay Freeport und der Clark Special Economic Zone abgemildert werden.

\subsection{Langfristiges Entwicklungsszenario (20-50 Jahre)}

a) Clark Air Base soll Standort eines neuen internationalen Flughafens für Manila werden:

Die absehbare Überlastung des Ninoy Aquino International Airport in Manila - er liegt im dicht besiedelten Süden der Hauptstadtregion - führt schon heute zu einer Diskussion über den Standort eines neuen Flughafens. Eine in der Diskussion stehende Alternative ist das $80 \mathrm{~km}$ nördlich gelegene Clark. Doch der Vorteil eines weniger dicht bebauten Umfeldes wird mit einer schwerer herzustellenden Erreichbarkeit aus der Hauptstadtregion erkauft. Die Anbindung soll auf lange Sicht über die Wiederinbetriebnahme der Bahnstrecke zwischen Angeles City und Manila sichergestellt werden. Dieses Projekt soll über eine innerstädtische Verlängerung der Bahnstrecke in Metro Manila auch Makati, den heutigen CBD der philippinischen Hauptstadtregion, und die zukünftige "Global City», das ehemalige Militärcamp Fort Bonifacio, anbinden.

b) Die Clark Special Economic Zone und Subic Bay Freeport and Special Economic Zone werden zu großen eigenständigen Entlastungszentren entwikkelt:

Die Entwicklung soll in den sehr langfristig angelegten Visionen auf eine Entwicklung der beiden Standorte jeweils zu großen Dienstleistungs- und Finanzzentren abzielen. Diese Standorte sollen eine Ausstrahlungskraft für ganz Südostasien besitzen und zu einer Entlastung der Primatstadt Metro Manila beitragen. Die Konzepte für die Gestaltung dieser neuen Zentren reichen heute schon bis zur detaillierten Flächennutzungsplanung im Rahmen eines Masterplans für die zukünftigen Zentral- bereiche. Diese sehr ambitionierten Entwicklungsvisionen entstammen der optimistischen Aufbruchsstimmung der frühen 1990er Jahre. Die Wahrscheinlichkeit einer Realisierung ist heute eher kritisch zu bewerten, doch haben diese Visionen schon jetzt Einfluss auf die Geschäftspraxis der BCDA-Töchter. Die Mietverträge mit Investoren werden gerade in den Zentralbereichen der Liegenschaften nur auf eine Laufzeit von 10 bis 20 Jahre abgeschlossen, was der langfristigen Entwicklung stabiler wirtschaftlicher Verhältnisse aufgrund fehlender Investitionssicherheit abträglich ist.Viele Unternehmensansiedlungen in den Zentralbereichen sind nur in Form einer $Z$ wischennutzung bestehender, mit relativ wenig Aufwand instandgesetzter Gebäudestrukturen möglich.

\section{Stand des Konversionsprozesses}

Der heutige Stand des Konversionsprozesses ist noch weit von diesen mittel- und langfristigen Zielen entfernt, doch ist festzustellen, dass in Subic und Clark Anfang 2000 wieder rund 50.000 Menschen Arbeit auf den ehemaligen Stützpunkten gefunden haben. Damit finden heute gut dreieinhalbmal so viele Personen eine Arbeit in den ehemaligen Militärstützpunkten wie kurze Zeit vor der Aufgabe der Stützpunkte.

Kleinere, kapitalschwächere Unternehmen nutzen in den Zentralbereichen alte Lagerhallen und einfache Mannschaftsunterkünfte. Entsprechend der Ausstattung dieser Gebäude findet man hier eher Betriebe, die in technologisch einfacheren Bereichen tätig sind (insbesondere Textilindustrie). Doch nicht nur diese, sondern auch größere Unternehmen internationaler Herkunft, bedienen sich bestehender Gebäudestrukturen. In den größeren, etwas außerhalb der Zentralbereiche gelegenen Gebäuden wurden hauptsächlich Produktionsstätten für technologisch weniger anspruchsvolle Elektronikartikel eingerichtet (z.B.Thomson produzierte kabelgebundene Telefone in Subic; siehe Abb. 2).

Doch daneben wurden im Rahmen von joint ventures mit japanischen und koreanischen Projektentwicklern außerhalb der Zentralbereiche Freiflächen für Industrieparks erschlossen. In diesen Bereichen befinden sich die wenigen größeren Betriebe, häufig der IT- und Unterhaltungselektronikbranche.

Ein Vergleich der beiden großen «Konversions-Ecozones» untereinander sowie ein Vergleich mit den «traditionellen» Freihandelszonen der PEZA verdeutlicht Gemeinsamkeiten und Unterschiede (Tab. 2).

Die beiden lokalen Entwicklungsgesellschaften Clark Development Corperation (CDC) und Subic Bay 


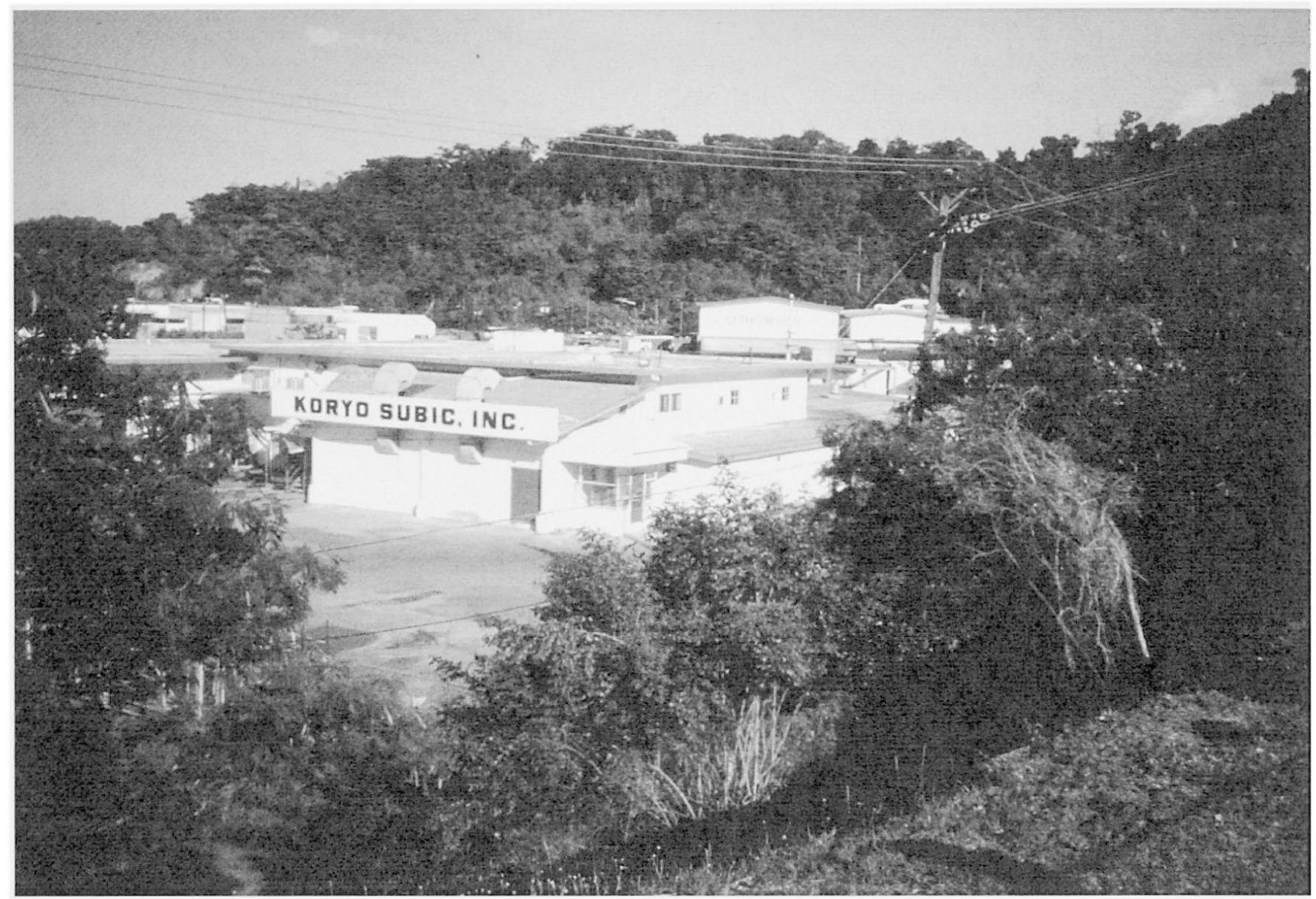

Abb. 2: Nachnutzung ehemaliger militärischer Gebäude in der Subic Bay Freeport Zone

Use of former military buildings in the Subic Bay Freeport Zone

Usages d'affectation d'anciens bâtiments militaires dans la "Subic Bay Freeport Zone»

Foto: V. ZIMmER, Januar 2001

Metropolitan Authority (SBMA) in den beiden «Konversions-Ecozones» haben sieben Jahre nach Beginn der Konversion im Jahr 1999 mit über 50.000 Arbeitsplätzen mehr Beschäftigungsmöglichkeiten geschaffen, als die US-Streitkräfte jemals direkt beschäftigt hatten. Obwohl die Zahl der Beschäftigten in beiden Freihandelszonen in einer ähnlichen Größenordnung liegt, unterscheiden sie sich doch in ihrem Exportvolumen erheblich: Die Subic Bay Freeport Zone exportiert Waren und Dienstleistungen in einem doppelt so hohen Wert wie die Clark Special Economic Zone. Das Exportvolumen pro Arbeitnehmer in Subic ist entsprechend rund dreimal höher als in Clark.

Die Analyse der Datenbestände der Labor Services Departments der BCDA-Töchter SBMA und CDC gibt hier einen Hinweis auf eine mögliche Erklärung: Subic konnte mehr Unternehmen der eher exportwertstärkeren Industriebereiche - wie die IT und Unterhaltungselektronikbranche - als Investoren ge- winnen. Rund $30 \%$ der Beschäftigten arbeiten in Betrieben dieser Branche. In Clark dagegen sind es nur $13 \%$ der Beschäftigten. Dagegen sind weniger exportwertstarke Zweige des produzierenden Gewerbes wie die Textilproduktion in Clark mit $25 \%$ der Beschäftigten weit stärker vertreten als in Subic mit nur $10 \%$.

Die «traditionellen» PEZA-Ecozones sind dagegen meist stark spezialisiert. Dieses wird darin deutlich, dass nur rund die Hälfte der 29 Ecozones mehr als zehn Betriebe beherbergen. Vergleicht man die Zahlen aller Freihandelszonen der PEZA mit denen der «Konversions-Ecozones», so werden die Unterschiede sehr deutlich: Die durchschnittliche Beschäftigtenzahl der Unternehmen in den PEZA-Ecozones ist weitaus größer als die der «Konversions-Ecozones», was die Vermutung nahe legt, dass hier kapitalstärkere Großunternehmen angesiedelt sind. Dieses wird gestützt durch die Tatsache, dass die Zahl der Unter- 


\begin{tabular}{|c|c|c|c|c|c|c|}
\hline & $\begin{array}{l}\text { Anzahl der } \\
\text { Unternehmen }\end{array}$ & $\begin{array}{l}\text { Arbeit- } \\
\text { nehmer }\end{array}$ & $\begin{array}{l}\text { Export- } \\
\text { volumen }\end{array}$ & \begin{tabular}{|l|} 
Durchschnittliche \\
Unternehmensgrösse
\end{tabular} & $\begin{array}{l}\text { Exportvolumen } \\
\text { pro Mitarbeiter }\end{array}$ & $\begin{array}{l}\text { Exportvolumen } \\
\text { pro } \\
\text { Unternehmen }\end{array}$ \\
\hline & & & Mio. US\$ & Arbeitnehmer & US\$ & Mio. US\$ \\
\hline $\begin{array}{l}\text { Subic Bay } \\
\text { Freeport } \\
\text { Zone }\end{array}$ & 340 & 20.200 & 1.100 & 59 & 54.500 & 3,2 \\
\hline $\begin{array}{l}\text { Clark Special } \\
\text { Economic } \\
\text { Zone }\end{array}$ & 188 & 29.900 & 505 & 159 & 16.900 & 2,7 \\
\hline $\begin{array}{l}29 \text { PEZA- } \\
\text { Ecozones }\end{array}$ & 704 & 617.700 & 15.800 & 877 & 25.600 & 22,4 \\
\hline
\end{tabular}

Tab. 2: «Konversions-Ecozones» im Vergleich (1999)

Conversion Ecozones in comparison (1999)

«Ecozones de conversion» comparées (1999)

Quelle: eigene Berechnung nach Daten des Labor Services Department der Clark Development Corporation (CDC), Bases Conversion and Development Authority (2000), Philippine Ecozone Authority (2000)

nehmen mit philippinischer Beteiligung in den PEZAEcozones mit nur $21 \%$ relativ klein ist; die Investoren stammen vornehmlich aus Japan, den USA und Korea. In den «Konversions-Ecozones» dagegen befinden sich 1999 zwischen 57\% (Subic) und 63\% (Clark) der Unternehmen zumindest zum Teil in philippinischer Hand. Es ist zu vermuten, dass es die vorhandene bauliche Struktur auf den Konversionsliegenschaften auch kapitalschwächeren, philippinischen Investoren ermöglicht, sich in einer Freihandelszone anzusiedeln. Damit haben es die «Konversions-Ecozones» geschafft, ein anderes (zusätzliches) Investorensegment zu erschließen. Die besondere Dynamik in den «Konversions-Ecozones» wird deutlich, wenn man sich die Entwicklung der Beschäftigtenzahlen zwischen 1997 und 1999 vor Augen hält: In Clark hat sich die Zahl mehr als verdoppelt, in Subic ist sie um ein Drittel größer geworden, wogegen die Beschäftigtenzahl in den traditionellen Ecozones sich nur um 10\% erhöhte. In diesen zwei Jahren hat sich das Exportvolumen der traditionellen Freihandelszonen nur um 36\% gesteigert, wogegen in Subic eine Verdoppelung $(+107 \%)$ und in Clark mehr als eine Verdreifachung $(+236 \%)$ registriert werden konnte.

\section{Stabilität des Konversionsprozesses}

Doch die Stetigkeit des Wachstums und die Stabilität des Erreichten sind kritisch zu bewerten. Immer wieder hat es Rückschläge gegeben. Große Investoren haben sich aus den "Konversions-Ecozones» zurückgezogen oder haben ihre Produktion gedrosselt. Thomson, einer der ersten großen Investoren in der Subic Freeport Zone mit zuletzt 900 Mitarbeitern, verlagerte seine gesamte Produktion kabelgebundener Telefone im Januar 2000 in die Volksrepublik China. Das Unternehmen folgte damit Erfordernissen, die aus dem immensen Preisverfall dieser technologisch einfachen Geräte resultierte. Damit zog sich ein Unternehmen zurück, das seine Produktion bewusst kostengünstig mittels Nutzung hinterlassener Militärgebäude an diesem Standort aufgebaut hatte (siehe Abb. 2). Eine geringe Investition macht Unternehmen bei Entscheidungen über die Wahl und den Wechsel eines Standortes sehr flexibel. Der Vorteil, in den «Konversions-Ecozones» eine fertige, mit geringem Aufwand anzupassende Infrastruktur vorzufinden, kehrt sich hier in einen gefährlichen Nachteil um.

Doch auch Unternehmen, die für ihre Standorte in den Industrieparks einen relativ großen Anteil der Gesamtinvestition in den Neubau einer Fabrikhalle gesteckt haben, sind $\mathrm{z} . \mathrm{Zt}$. verstärkt versucht, den noch günstigeren Produktionsbedingungen in chinesischen Freihandelszonen zu folgen. So hat ein anderer «Premiuminvestor», der taiwanesische Computerhersteller Acer, Teile seiner Computer-Fertigung, die nach dem Erdbeben im Heimatland Taiwan in Subic ausgebaut wurde, inzwischen nach China verlegt.

Die «Konversions-Ecozones» werden zukünftig um ihre Marktposition unter den Freihandelzonen der Welt kämpfen müssen. Denn die Vorteile, wie die englische Sprachkompetenz der Arbeitskräfte und die vorhandene Infrastruktur, werden nicht dauerhaft die sehr viel günstigeren Produktionsbedingungen in ande- 
ren Freihandelszonen übertreffen können. Strukturelle Unterschiede werden schon deutlich in der Tatsache, dass in den Freihandelszonen der Welt aufgrund der niedrigeren Personalkosten fast ausschließlich Frauen arbeiten (90\%) (INTERNATIONAL Labour ORGanisaTION 1998, zit. nach KNOX \& MarsTon 2001: 350). In den «Konversions-Ecozones» ist das Verhältnis zwischen den Geschlechtern dagegen zahlenmäßig fast ausgeglichen.

\section{Fazit}

Die BCDA und ihre örtlichen Tochterunternehmen haben es verstanden, kurzfristig unter Nutzung vorhandener Ressourcen ein Angebot für Investitionen zu schaffen, das es ermöglicht, bereits jetzt wieder mehr Leute auf den ehemaligen Stützpunkten zu beschäftigen als zuletzt bei den US-Streitkräften und mit 500 Unternehmen rund 3\% der philippinischen Exporterlöse zu erwirtschaften. Doch erweist sich die Entwicklung als nicht stabil. Konkurrenzstandorte im geöffneten Wirtschaftsraum Chinas stellen gerade für Unternehmen, die mit einem relativ geringen Investitionsvolumen ihre Produktion in Subic und Clark aufgebaut haben, eine attraktive Alternative dar. Die Umsetzbarkeit der mittel- und langfristigen Visionen kann heute noch nicht bewertet werden. Eine in diesen Visionen vorgezeichnete Entwicklung zu starken Zentren könnte eine Entlastung der Primatstadt Manila bedeuten, doch ist gerade vor dem Hintergrund der Gefahr eines möglichen Abbruchs der Investitionstätigkeit eine allzu optimistische Bewertung nicht angebracht.

\section{Literatur}

Bases Conversion and Development Authority (2000): Annual Report 1999. - Manila: Selbstverlag. Biehler, H., Richter, G. \& D. Sträter (1990): Regionale Konversionsforschung. Bestandsaufnahme und Handlungsorientierung. - In: KölLnER, L. \& B. HucK (Hrsg.): Abrüstung und Konversion. Politische Voraussetzungen und wirtschaftliche Folgen in der Bundesrepublik. $-=$ Reihe des Instituts für Medienforschung und Urbanistik 1, Frankfurt, New York: 433-490.

Bonn International Center for Conversion (2000): Conversion Survey 2001. Global Disarmament, Demilitarization and Demobilization. - Baden-Baden: Nomos. Bronger, D. (1987): Die Philippinen: Raumstrukturen, Entwicklungsprobleme, regionale Entwicklungsplanung. $-=$ Mitteilungen des Instituts für Asienkunde 159, Hamburg: Selbstverlag.

Carpenter, T. (1991): The U.S. Military Presence in the Philippines: Expensive and Unnecessary. - In: Foreign Policy Briefing 12, http://www.cato.org/pubs/fpbriefs/ fpb-012.html, 30.3.2000.

Cranston, A. (1990): Economic Growth Vital to Phil- ippine Democracy. Comments before the Senate, May 11. - In: Congressional Record, May 11 1990, Tracking No. 140249.

Department of Defense (1990): Total Civilian Personnel Strengths by Regional Area and by Country. Washington: Selbstverlag.

INTERNATIONAL LABOUR ORGANISATION (1998): Labour and Social Issues Relating to Export Processing Zones. Report for discussion at the Tripartite Meeting of Export Processing Zones-Operating Countries, Geneva, 1998. - Geneva: Selbstverlag.

JoRSTAD, R., Statistical Information Analysis Division, Department of Defense, Persönliche Mitteilung im März 2001.

KLÜVER, H. (1987): Bundeswehrstandorte im ländlichen Raum. Wirtschaftsgeographische Auswirkungen der Garnisonen Diepholz und Stadtallendorf. - = Marburger Geographische Schriften 107, Marburg: Selbstverlag.

KNox, P. \& S. Marston (2001): Humangeographie. Heidelberg, Berlin: Spektrum Akademischer Verlag. MenKe-GlückerT, P. (1991): Rückrüstung. Eine Aufgabe für Entsorger. - In: Umwelt 21,4: 194-203.

Philippine Economic Zone Authority (2000): Performance Indicators. - http://www.philippines1.com/ peza/pi.html, 22.04.2000.

Rocher, S. DU (1990): Les bases américaines aux Philippines: Enjeux et perspectives. $-=$ Fondation pour les études de défense nationale, Dossier 35, Paris: Selbstverlag.

RoEDER, U. (1989): Auswirkungen von Bundeswehrgarnisonen auf die regionale Wirtschaftsstruktur. = Nürnberger Wirtschafts- und Sozialgeographische Arbeiten 41, Nürnberg: Selbstverlag.

Statistisches Bundesamt (1993): Länderbericht Philippinen 1992. - Kusterdingen: Verlag J.B. Metzler und C.E. Poeschel.

WinKELMANN, P. (1995): Konversionsmanagement von ehemaligen militärischen Liegenschaften. Kooperationsmodelle zwischen öffentlichem und privatem Sektor bei Konversionsprojekten. - = Schriftenreihe des Instituts für Umweltschutz der Universität Dortmund 28, Dortmund: Selbstverlag.

\section{Zusammenfassung: Konversion der US-Stützpunkte auf den Philippinen: Kompensationsstrategien in militärdominierten Regionalökonomien}

Der sehr kurzfristige Abzug der US-Streitkräfte im Jahr 1992 versetzte die Philippinen in die Situation, den Verlust von rund 14.000 Arbeitsplätzen für einheimische Beschäftigte und ein Ausgabevolumen der USArmee von jährlich mehr als 700 Mio. US\$ kompensieren zu müssen. Die gut ausgebaute Infrastruktur der verlassenen US-Stützpunkte sowie die Finanzierung über einen revolvierenden Fonds, dessen Erlöse sich aus der Entwicklung und dem Verkauf großer Militärareale der philippinischen Streitkräfte in der 
Hauptstadtregion generieren, haben einen zügigen Beginn der Liegenschaftskonversion ermöglicht. Die Zahl der heute in den Konversions-Ecozones geschaffenen neuen Arbeitsplätze übersteigt mittlerweile die Beschäftigung zu Zeiten der US-Streitkräfte bei weitem. Trotzdem bleibt die Entwicklung noch weit von den visionären Vorstellungen der anfänglichen Euphorie entfernt und die Stabilität der heutigen Branchenstruktur ist kritisch zu bewerten. Ein Vergleich der «Konversions-Ecozones» mit den anderen philippinischen Freihandelszonen zeigt jedoch auch, dass diese grundlegend anders strukturiert sind und insbesondere auch kleinere, philippinische Unternehmen in den «Konversions-Ecozones» eine Zugangsmöglichkeit zu einer Freihandelszone finden.

\section{Summary: Conversion of the US-Bases in the Philip- pines: Strategies of compensation in regional econo- mies dominated by the military}

The sudden withdrawal of the US-armed forces in 1992 compelled the Philippines to find sustainable solutions to compensate the loss of about 14.000 jobs for domestic employees and an annual volume of spending of about 700 Mio. US\$ by the US-forces. The well-developed infrastructure of the left bases and the option to finance the investments by a revolving fund made it possible to start the conversion of the bases in a very short period of time. The revolving fund generated its proceeds by the development and sale of military bases in the National Capital Region, formerly used by the Philippine armed forces. Today, more jobs have been created in the former US-bases than had been ever provided by the US-forces at any time. Nevertheless the development of the former bases is still far away from the optimistic ideas envisioned in the very first years of the conversion-process and the stability of the development has to be judged critically as well. A comparison between the "conversion-ecozones» and the other Philippine special economic zones shows that there are basic differences between these zones, in particular regarding the accessibility awarded to small, Philippine owned entrepreneurs (which is made possible in the conversion zones).

\section{Résumé: Reconversion des bases américaines aux Philippines: stratégies de compensation pour les économies régionales placées sous la domination militaire}

Aux Philippines, le départ à très court terme des troupes américaines, en 1992, a provoqué une situation de perte d'emploi pour 14000 employés résidents et l'obligation de compenser un budget de dépense de l'armée des Etats-Unis, de plus de 700 millions de dollars par an. L'infrastructure bien développée des bases militaires abandonnées et le financement par un fonds renouvelable, dont les revenus se génèrent à partir du développement et de la vente d'importants terrains militaires de l'armée des Philippines dans la région de la capitale, ont permis d'entreprendre rapidement la conversion des terrains et de l'immobilier. Le nombre des nouveaux emplois créés dans les «zones économiques de conversion» dépasse à présent largement celui du temps de la présence américaine. Malgré tout, le développement reste très en deçà des prévisions de l'euphorie visionnaire des origines, et la stabilité de la structure actuelle des secteurs économiques reste critique. Une comparaison des «zones économiques de conversion" avec d'autres zones de libre-échange montre aussi qu'elles possèdent des structures très différentes et qu'elles permettent notamment aux petites entreprises des Philippines d'avoir accès à une telle zone d'activité.

\section{Didaktische Hinweise}

- a. Erläutern Sie bitte das im Vorfeld des Abzuges der US-Streitkräfte diskutierte Für und Wider einer Stationierung auf den Philippinen aus den verschiedenen Perspektiven.

b. Im Jahr 1999 wurde zwischen den Philippinen und den USA das Visiting Forces Agreement (VFA) geschlossen. Es enthält Regelungen über gegenseitige Truppenbesuche und gemeinsame Übungen der Streitkräfte beider Staaten. Von Kritikern wird es als Vorstufe eines neuen Stationierungsabkommens mit den USA gesehen. Informieren Sie sich im Internet über die damalige Diskussion und bewerten Sie insbesondere die Anwendung des VFAs nach dem 11. September 2001.

- Erläutern Sie die Bedeutung eines «revolvierenden Fonds». Suchen Sie nach anderen Beispielen, bei denen dieses Instrument der Finanzierung zum Einsatz kommt.

- Welche Erfolge brachte nach Abzug des Militärs die zivile Umnutzung?

- Welche Faktoren könnten die heute stabile Lage in Zukunft schwächen?

- Vergleichen Sie die philippinischen «KonversionsEcozones» mit anderen Economic Zones auf den Philippinen und in Südostasien. Was sind die Unterschiede und in welcher Form konnten diese als Vorteil genutzt werden? Wodurch ist eine stabile Entwicklung in den philippinischen «Konversions-Ecozones» gefährdet?

Volker Zimmer, Institut für Geographie, Universität Hamburg, Bundesstraße 55, D-20146 Hamburg. e-mail:zimmer@geowiss.uni-hamburg.de

\section{Manuskripteingang/received/manuscrit entré le 27.2.2002}

Annahme zum Druck/accepted for publication/accepté pour l'impression: 16.2.2004 